\title{
Malestar psicofisiológico en profesionales de la salud pública de la Región Metropolitana
}

\author{
ELISA ANSOLEAGA ${ }^{1, \mathrm{a}, \mathrm{b}}$, JUAN PABLO TORO C. ${ }^{1, \mathrm{a}}$, LORENA GODOY C., ${ }^{1,2, \mathrm{c}}$, \\ ANTONIO STECHER ${ }^{1,3, a}$, JOSEP M. BLANCH ${ }^{3, a}$
}

\section{Psychophysiological distress among health care professionals working in Chilean public hospitals}

Background: The public health reform in Chile resulted in changes in working conditions and organization of health centers. Aim: To examine the presence of psychophysiological symptoms in professionals of public hospitals in the Metropolitan Region and their association with current working conditions. Material and Methods: A questionnaire of quality of working life was applied to a sample of 80 physicians and 110 nurses. The questionnaire considers scales and open questions. Results: Nurses had a higher level of discomfort than physicians $(p<0.01)$ and had significantly higher scores for emotional distress, physical fatigue, digestive disorders, headache, insomnia, back pain and muscle tension $(p<0.01)$. There were statistically significant negative correlations between psychophysiological distress and working conditions $(r=-0.418)$, social climate $(r=-0.395)$, satisfaction with the organization $(r=-0.337)$ and psychosocial well-being $(r=-0.267)$. A regression model showed that $21 \%$ of the variance in psychophysiological distress was explained by working conditions, psychosocial well-being and adaptation to the organization. Conclusions: There is a relationship between the high prevalence of psychophysiological symptoms and bad working conditions of public health professionals.

(Rev Med Chile 2011; 139: 1185-1191).

Key words: Psychology, social; Sociology; Women, working.

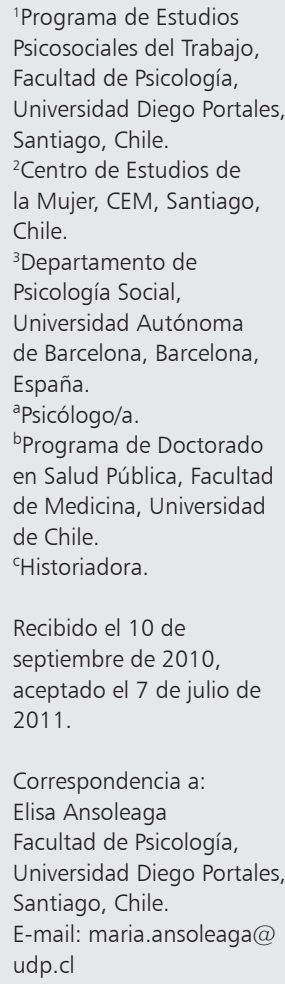

Correspondencia a:

Elisa Ansoleaga

Facultad de Psicología Universidad Diego Portales, Santiago, Chile.

E-mail: maria.ansoleaga@ udp.cl

L a reforma y modernización de los sistemas de salud pública, orientadas por el paraJigma de la Nueva Gestión Pública, han caracterizado a la realidad de muchos países de América Latina a partir de la década $1980-89^{1,2}$. Este proceso de reforma ha significado que la red de instituciones de salud pública, tradicionalmente autónoma con respecto a los principios del mercado, se haya reorganizado gradualmente de acuerdo con un nuevo modelo de gestión, denominado capitalismo sanitario ${ }^{3}$ y una lógica caracterizada, entre otros, por: tendencia a la fragmentación y descentralización organizativa; fuerte énfasis en la reducción de los costos y aumento de la eficiencia; introducción de mecanismos de competencia entre los diferentes proveedores públicos y privados; uso más masivo y sistemático de formas flexibles de empleo; externalización de diversas funciones a empresas privadas; nuevos sistemas de seguimiento y evaluación centrados en el rendimiento individual y formas flexibles de gestión de recursos humanos basados en incentivos con objetivos e indicadores de desempeño medibles.

En Chile la reforma de salud ha implicado significativos cambios en la experiencia de trabajo de sus profesionales ${ }^{4,5}$, la que, sin embargo, ha recibido escasa atención. Existe bastante acuerdo en declarar el rol fundamental que tiene el recurso humano en el marco de estas reformas, al cual incluso se le reconoce como una ventaja competitiva ${ }^{6}$. No obstante, este discurso no se ve plasmado efectivamente en prácticas, políticas 
y programas que le asignen tal valor. "Lo cierto es que las cuestiones del personal han estado ausentes de las agendas políticas de las reformas del sector salud en casi todos los países de la región..."(6 p1).

Sin embargo, se constata un incremento de la literatura referida a la experiencia de trabajo, las relaciones entre las actuales condiciones de trabajo, el bienestar, la salud y la calidad de vida en profesionales de la salud. A nivel nacional cabe destacar los aportes realizados por Horwitz ${ }^{7}$ respecto a los desafíos psicosociales de la práctica médica, el estudio de Jiménez, JP ${ }^{4}$ sobre bienestar y satisfacción en médicos en el contexto de la refor$\mathrm{ma}$, el de Schönhaut y $\mathrm{col}^{8}$ sobre calidad de vida en médicos residentes y los estudios sobre estrés ocupacional en personal de salud de Trucco y col', entre otros. Asimismo existe una vasta literatura en el ámbito de enfermería, entre otros, los estudios de Rodríguez y col $^{10}$ sobre burnout, los de Astudillo $\mathrm{y} \mathrm{col}{ }^{11}$ sobre factores protectores de estrés laboral y el estudio de Avendaño y col ${ }^{12}$ sobre burnout y apoyo social en personal del servicio de psiquiatría de un hospital público.

Los estudios nacionales son coincidentes con hallazgos a nivel internacional revelando que médicos y enfermeras del sector público tienen mayores niveles de distrés ${ }^{13}$ al compararlos con profesionales del sector privado ${ }^{14,15}$, y con población general ${ }^{16}$.

Entonces, parece necesario revalorizar el importante rol que pueden jugar las personas en la implementación de los procesos de reforma. En este contexto, la investigación iberoamericana Proyecto Capitalismo Organizacional como Factor de Riesgo Psicosocial ${ }^{17}$ (en adelante Kofarips), en la cual se enmarca este trabajo, tuvo como objetivo estudiar el impacto psicosocial de las actuales condiciones laborales en las experiencias del personal médico y de enfermería en hospitales públicos. El propósito particular de este artículo es examinar la presencia de sintomatología psicofisiológica en profesionales de la salud pública y sus asociaciones con las actuales condiciones de trabajo.

\section{Material y Método}

\section{Tipo de estudio}

Transversal descriptivo, asociativo y correlacional con carácter exploratorio.

\section{Muestra}

No probabilística y ocasional. Consideró 80 médicos y 110 enfermeras, trabajadores de 10 hospitales públicos de la Región Metropolitana, siete de ellos corresponden a establecimientos de mayor complejidad y tres a establecimientos de mediana complejidad según la categorización del Ministerio de Salud de Chile. Los participantes fueron contactados por vías institucionales y por contactos personales. El promedio de edad para médicos fue 46 años y para enfermeras 39 años. La mayoría de los profesionales médicos trabaja a tiempo parcial $(72 \%)$ mientras que en profesionales de enfermería la mayoría trabaja a tiempo completo (91\%). En los profesionales médicos, se observa una mayor proporción de hombres (61\%) mientras que en profesionales de enfermería existe una proporción significativamente mayor de mujeres (95\%). Respecto de la muestra total $71 \%$ son mujeres y $29 \%$ hombres coincidiendo con la distribución general de profesionales de la salud en Chile. La distribución de los datos no hizo posible realizar análisis estratificados conjuntos por sexo y profesión.

\section{Instrumento}

Se aplicó el cuestionario de Calidad de Vida Laboral (Tabla 1) desarrollado por el proyecto Kofarips que consta de 10 escalas tipo Likert con distinto tramo de respuesta, un inventario y 15 preguntas de respuesta abierta. Este trabajo consideró 7 escalas. Las escalas 1, 2, y 6 son adaptaciones de instrumentos utilizados en los estudios: "Investigación Iberoamericana sobre Calidad de Vida Laboral en el Subempleo"18 y "Calidad de Vida Laboral en Hospitales"19, y las escalas 3, 4, 5 y 7 fueron diseñadas específicamente para la investigación Kofarips, previa revisión de literatura, discusión de grupos de expertos y un estudio piloto realizado a finales de 2008 ( $\mathrm{n}=220)$ que permitió mejorar las características psicométricas del instrumento, posteriormente revalidado a partir de su aplicación en 2009 a una muestra iberoamericana $(\mathrm{n}=1.252)^{20,21}$.

El instrumento se autoadministró entre enero y agosto de 2009. Contó con el consentimiento informado y voluntariedad, expresa y por escrito, de los participantes.

\section{Análisis estadísticos}

Se realizaron análisis de estadística descriptiva e inferencial (diferencias de medias, ANOVAS, co- 
Tabla 1. Escalas del instrumento KOFARIPS, confiabilidad y definición de conceptos que considera cada una

\begin{tabular}{|c|c|c|c|c|}
\hline Escalas & $\begin{array}{l}\text { Alpha } \\
\text { Cronbach* }\end{array}$ & $\begin{array}{c}\text { Alpha } \\
\text { Cronbach } \\
\text { escalas Chile }\end{array}$ & $\begin{array}{c}n \\
\text { ítems }\end{array}$ & Definición \\
\hline $\begin{array}{l}\text { 1. Condiciones de Trabajo } \\
(0=\text { pésimo / } 10=\text { óptimo })\end{array}$ & 0,90 & 0,91 & 14 & $\begin{array}{l}\text { Propiedades (ambientales, materiales, } \\
\text { técnicas, salariales, contractuales, or- } \\
\text { ganizacionales, etc) percibidas del en- } \\
\text { torno objetivo de la actividad laboral }\end{array}$ \\
\hline $\begin{array}{l}\text { 2. Clima Social de Trabajo } \\
(0=\text { pésimo } / 10=\text { óptimo })\end{array}$ & 0,87 & 0,89 & 8 & $\begin{array}{l}\text { Características percibidas del ambiente } \\
\text { sociolaboral }\end{array}$ \\
\hline $\begin{array}{l}\text { 3. Satisfacción con la Organización } \\
\text { (1=tot desacuerdo / } 7=\text { tot acuerdo) }\end{array}$ & 0,95 & 0,93 & 10 & $\begin{array}{l}\text { Satisfacción personal por la actual } \\
\text { organización del trabajo en el propio } \\
\text { centro }\end{array}$ \\
\hline $\begin{array}{l}\text { 4. Adaptación a la Organización } \\
\text { (1=tot desacuerdo / } 7=\text { tot acuerdo) }\end{array}$ & 0,89 & 0,88 & 7 & $\begin{array}{l}\text { Niveles de aceptación y acatamiento a } \\
\text { la misión, política y formas de organi- } \\
\text { zación promovidas por la dirección del } \\
\text { centro hospitalario }\end{array}$ \\
\hline $\begin{array}{l}\text { 5. Ideología Mercantilista } \\
\text { (1=tot desacuerdo / } 7=\text { tot acuerdo) }\end{array}$ & 0,86 & 0,83 & 12 & $\begin{array}{l}\text { Grado de acuerdo con el enfoque } \\
\text { empresarial, mercantil y managerial } \\
\text { en el diseño y la gestión de un servicio } \\
\text { público }\end{array}$ \\
\hline $\begin{array}{l}\text { 6. Bienestar Psicosocial } \\
\text { ( } 1=\text { negativo } / 7=\text { positivo })\end{array}$ & 0,95 & 0,96 & 16 & $\begin{array}{l}\text { Nivel de bienestar percibido en la ac- } \\
\text { tual experiencia de trabajo }\end{array}$ \\
\hline $\begin{array}{l}\text { 7. Malestar Psicofisiológico } \\
(1=\text { nunca } / 7=\text { siempre })\end{array}$ & 0,90 & 0,89 & 12 & $\begin{array}{l}\text { Efectos psicosomáticos colaterales } \\
\text { percibidos como asociados a la propia } \\
\text { experiencia actual de trabajo }\end{array}$ \\
\hline
\end{tabular}

* (A partir de los 1.020 primeros cuestionarios implementados en la base de datos Kofarips, básicamente provenientes de España, Brasil, Chile y Colombia). 07122009.

rrelaciones y regresión lineal múltiple), se trabajó con un nivel de significación $95 \%$.

El estudio se enfocó principalmente en la variable malestar psicofisiológico atendiendo a las puntuaciones en la escala correspondiente, que abarca un conjunto de síntomas físicos, psicológicos y psicosomáticos atribuidos a la experiencia laboral: desgaste emocional, agotamiento físico, saturación mental, mal humor, sentimiento de baja realización profesional, trato despersonalizado a colegas o usuarios, frustración, trastornos digestivos, dolor de cabeza, insomnio, dolor de espalda y tensión muscular.

A través de un Contraste de Levene sobre la igualdad del error de las varianzas, se estudió la asociación entre malestar psicofisiológico y una serie de variables sociodemográficas contempladas por el cuestionario. Asimismo, fueron analizadas las correlaciones $(r)$ entre las puntuaciones medias en la variable malestar psicofisiológico y las de otras variables del cuestionario: condiciones de trabajo, clima social de trabajo, satisfacción con la organización, adaptación a la organización, ideología mercantilista y bienestar psicosocial (Tabla 1). Finalmente, a fin de identificar las variables predictoras del malestar psicofisiológico se realizó una regresión múltiple, utilizando la técnica del stepwise. Los datos fueron procesados mediante el software SPSS 17.0.

\section{Resultados}

En primer lugar, un análisis de los ítems de la escala de malestar psicofisiológico revela una importante prevalencia de sintomatología en profesionales de la medicina y de la enfermería al indicar la frecuencia con que su trabajo les ocasiona los siguientes síntomas (Tabla 2). Asimismo 
los profesionales de enfermería muestran puntajes más altos y estadísticamente significativos en desgaste emocional $(\mathrm{p}=0,041)$, agotamiento físico $(\mathrm{p}=0,003)$, trastornos digestivos $(\mathrm{p}=0,000)$, dolores de cabeza $(p=0,000)$, insomnio $(p=0,000)$, dolores de espalda $(\mathrm{p}=0,000)$ y tensión muscular $(\mathrm{p}=0,000)($ Tabla 3$)$.

El análisis de las asociaciones entre la escala de malestar psicofisiológico y los datos sociodemográficos y laborales descubre diferencias significativas según profesión $(\mathrm{p}=0,000)$ : los profesionales de enfermería presentan un mayor nivel de malestar. Asimismo se aprecian diferencias significativas según dedicación laboral al centro $(p=0,002)$ : quienes tienen jornada completa presentan un nivel más elevado de malestar. Este dato es coherente con el anterior, ya que la mayoría del personal de enfermería trabaja a tiempo completo a diferencia del de medicina, que suele trabajar a tiempo parcial en su centro. No se observan otras asociaciones estadísticamente significativas.

También surgen correlaciones negativas y significativas, aunque de variada intensidad, entre las puntuaciones en la escala de malestar psicofisiológico y las de condiciones de trabajo $(r:-0,418$ $\mathrm{p}=0,000)$, clima social $(r:-0,395 \mathrm{p}=0,000)$, satisfacción con la organización $(r:-0,337 \mathrm{p}=0,000) \mathrm{y}$ bienestar psicosocial $(r:-0,267 \mathrm{p}=0,000)$.

Finalmente, se estudió la relación lineal entre la variable malestar psicofisiológico y el resto de las variables del estudio a través de una regresión múltiple. Utilizando la técnica del stepwise, se generaron tres modelos, de los cuales el que explica la mayor cantidad de varianza incluye las variables: condiciones de trabajo, bienestar psicosocial y adaptación a la organización (Tabla 4). El valor del coeficiente de correlación múltiple $(\mathrm{R}=0,46)$ denota de una moderada magnitud y señala que las condiciones de trabajo, el bienestar psicosocial y la adaptación a la organización explican el $21 \%$ de la varianza en malestar psicofisiológico $\left(\mathrm{R}^{2}=0,212\right.$; $\mathrm{R}^{2}$ ajustado $\left.=0,199 ; \mathrm{F}=3.995 ; \mathrm{p}=0,047\right)$. Además, los valores Beta $(\beta)$ revelan significancia estadística $[\beta=-0,425(\mathrm{p}=0,000)$ para condiciones de trabajo; $\beta=-0,188(\mathrm{p}=0,011)$ y para bienestar psicosocial y $\beta=0,153(\mathrm{p}=0,047)$ ] lo que refuerza la asociación entre malestar psicofisiológico y condiciones de la organización del trabajo.

Habiendo constatado que la más alta correlación se presentó entre peores condiciones de trabajo y malestar psicofisiológico, interesó profundizar
Tabla 2. Porcentaje de médicos y enfermeras que manifiestan haber tenido "siempre o casi siempre sintomatología asociada al trabajo"

\begin{tabular}{|lcc|}
\hline & $\begin{array}{c}\text { Médicos } \\
\text { (\%) }\end{array}$ & $\begin{array}{c}\text { Enfermeras } \\
\text { (\%) }\end{array}$ \\
\hline Desgaste emocional & 33 & 44 \\
\hline Agotamiento físico & 36 & 54 \\
\hline Saturación mental & 34 & 41 \\
\hline Mal humor & 13 & 11 \\
\hline $\begin{array}{l}\text { Sentimiento de baja } \\
\text { realización }\end{array}$ & 8 & 12 \\
\hline $\begin{array}{l}\text { Trato despersonalizado } \\
\text { a colegas o usuarios }\end{array}$ & 7 & 9 \\
\hline Frustración & 9 & 9 \\
\hline Trastornos digestivos & 5 & 23 \\
\hline Dolores de cabeza & 8 & 26 \\
\hline Insomnio & 8 & 16 \\
\hline Dolores de espalda & 9 & 23 \\
\hline Tensiones musculares & 13 & 42 \\
\hline
\end{tabular}

Tabla 3. Anova entre médicos y enfermeras para la escala de malestar psicofisiológico

\begin{tabular}{|lccc|}
\hline & $\begin{array}{c}\text { Promedio } \\
\text { medicina }\end{array}$ & $\begin{array}{c}\text { Promedio } \\
\text { enfermería }\end{array}$ & Sig. \\
\hline Desgaste emocional & 4,47 & 4,93 & 0,041 \\
\hline Agotamiento físico & 4,68 & 5,32 & 0,003 \\
\hline $\begin{array}{l}\text { Saturación mental } \\
\text { Mal humor }\end{array}$ & 4,50 & 4,83 & 0,203 \\
\hline $\begin{array}{l}\text { Sentimiento de } \\
\text { baja realización } \\
\text { profesional }\end{array}$ & 3,32 & 3,59 & 0,249 \\
\hline $\begin{array}{l}\text { Trato despersonali- } \\
\text { zado a colegas o a } \\
\text { personas usuarias } \\
\text { del servicio }\end{array}$ & 2,83 & 3,31 & 0,125 \\
\hline $\begin{array}{l}\text { Frustración } \\
\text { Trastornos diges- }\end{array}$ & 3,11 & 3,18 & 0,762 \\
tivos & 2,00 & 3,41 & 0,000 \\
\hline $\begin{array}{l}\text { Dolores de cabeza } \\
\text { Insomnio }\end{array}$ & 2,44 & 3,85 & 0,000 \\
\hline $\begin{array}{l}\text { Dolores de espalda } \\
\text { Tensiones muscu- }\end{array}$ & 2,07 & 3,27 & 0,000 \\
lares & 2,50 & 4,17 & 0,000 \\
\hline & 2,97 & 4,50 & 0,000 \\
\hline
\end{tabular}


Tabla 4. Malestar psicofisiológico: análisis de regresión múltiple considerando como predictivas las variables condiciones de trabajo, bienestar psicosocial y adaptación a la organización

\begin{tabular}{|lcccc|}
\hline Variable & B & $\beta$ & t & p \\
\hline Condiciones de trabajo & $-0,318$ & $-0,425$ & $-5,65$ & 0,000 \\
\hline Bienestar psicosocial & $-0,187$ & $-0,188$ & $-2,57$ & 0,011 \\
\hline Adaptación a la organización & 0,190 & 0,153 & 1,99 & 0,047 \\
\hline
\end{tabular}

el análisis, estudiando las respuestas abiertas del cuestionario que tratan específicamente de la relación entre condiciones y carga de trabajo. A ese respecto, más de la mitad de los participantes ( $51 \%$ de las enfermeras y $54 \%$ de los médicos) consideran que los aspectos técnicos, materiales, de infraestructura y de tecnología médica han presentado mejoras. Sin embargo, reportan asimismo una tendencia al incremento de la carga de trabajo. Enfermeras (27\%) y médicos $(16 \%)$ mencionan que "lo que ha empeorado en su trabajo" se relaciona con: la falta de tiempo para realizar sus tareas, la carga laboral y el aumento de la presión asistencial.

El incremento de la carga laboral es descrito en términos de la cantidad e intensidad de trabajo que les demanda la organización. Así, esta sobrecarga es asociada a un conjunto de consecuencias negativas para el ejercicio de la profesión: a) impacta en el trato y cuidado de los pacientes; b) repercute en las relaciones interpersonales entre colegas; c) genera sentimientos de estrés, cansancio, ansiedad, agobio y abatimiento. Lo anterior es reportado como un componente habitual de la experiencia de trabajo: $58 \%$ de las enfermeras y $41 \%$ de los médicos reportan que "siempre les falta tiempo" y sostienen que viven esta situación con una fuerte dosis de estrés cotidiano. Así como el incremento de la carga laboral tiene consecuencias negativas en el ámbito profesional, se reconoce también que la realización de horas extras y el llevarse trabajo para la casa tiene un impacto en la vida personal y familiar de los profesionales.

En el caso de las enfermeras, entre los elementos asociados a sobrecarga, relatan: una mayor cantidad de usuarios de los servicios, un tipo de usuario más demandante y asertivo, la exigencia por cumplir múltiples tareas simultáneamente (cuidado, registro, coordinación, etc), y unos recursos humanos no proporcionales al crecimiento de la carga de trabajo.

\section{Discusión}

Los resultados ponen de manifiesto una alta prevalencia en sintomatología psicofisiológica en el grupo de profesionales de la salud, más aún si se considera el sesgo del trabajador sano (este estudio se hizo en población potencialmente sana que se encontraba presente en su lugar de trabajo). Este resultado es consistente con los hallazgos reportados por Trucco ${ }^{9}$ en profesionales de la salud en general del ámbito nacional y con los de otros estudios en el plano internacional ${ }^{22,23}$. Los profesionales de enfermería reportan altos puntajes en las escalas de desgaste emocional, trato despersonalizado con colegas y usuarios, y baja realización personal, tres de los componentes del denominado síndrome de quemarse por el trabajo (burnout) que ha sido ampliamente estudiado en profesionales de la salud ${ }^{24-26}$. Similares tendencias son detectadas en personal médico ${ }^{27,28}$.

En un estudio en curso, realizado con población homóloga a la ésta, investigadores de la Universidad Autónoma de Barcelona (Blanch, Sahagún, Cantera y Cervantes, 2010) aplicaron conjuntamente la escala de malestar psicofisiológico y una versión española del Maslach Burnout Inventory $(\mathrm{MBI})^{29}$. Se observó que la escala de malestar psicofisiológico correlacionó alta y positivamente con los diversos factores de las escalas del MBI. Así, por ejemplo, el factor desgaste de la escala de malestar psicofisiológico (que incluye los ítems sobrecarga de trabajo, desgaste emocional, agotamiento físico y saturación mental) presenta una correlación de $\mathrm{r}=0,67(\mathrm{p}<0,001)$ con el factor Desgaste Emocional del MBI. La consistencia en los resultados de ambos instrumentos y los resultados de alta sintomatología aquí descritos, permiten asignar a esta población un alto riesgo de quemarse por el trabajo ${ }^{30}$.

Este estudio aporta en el reconocimiento de la sintomatología psicofisiológica asociada al trabajo, 
sin embargo, complementa la mirada poniendo su foco en las posibles relaciones entre esta sintomatología y los elementos de la organización del trabajo, particularmente, con las experiencias que los profesionales reportan a partir de las prácticas y valores de la gestión institucional.

De este modo, las enfermeras se aproximan más funcional y adaptativamente a las nuevas prácticas de gestión, presentan una mayor adhesión a las normas de la organización y a la ideología gerencialista, sin embargo, paradójicamente, presentan mayores niveles de malestar psicofisiológico y sobrecarga de trabajo. Esta aparente disonancia puede explicarse por un cambio cuantitativo en el trabajo de enfermería, en tanto su rol tradicional en la gestión de procesos administrativos, bajo la tendencia de la nueva gestión, supone un aumento de responsabilidades y tareas. También se puede hipotetizar que se trata de un cambio cualitativo, que las tareas de administración y gestión no han variado mayormente, sino que adquieren un estatus superior que aporta mayor reconocimiento, ante lo cual, se despliega mayor compromiso. En ambos casos, en la base de la experiencia de sobrecarga se aprecia una sobredemanda, cualitativa o cuantitativa, que redunda en mayor presencia de sintomatología.

Aun cuando profesionales de la medicina y de la enfermería difieren en los puntajes de sintomatología psicofisiológica, en ambos casos las cifras son preocupantes. Las diferencias encontradas pueden asociarse no sólo a la naturaleza de sus ocupaciones, sino también a los tipos de jornadas laborales. Mientras en enfermería se trabaja a tiempo completo en hospitales públicos, el personal médico lo hace predominantemente en jornada parcial, por lo cual presentaría un nivel de exposición diferente a las actuales condiciones de trabajo que son percibidas como patogénicas.

Entre las limitaciones de este estudio destaca su carácter exploratorio y la ausencia de representatividad de la muestra respecto de la población de profesionales de la salud pública en la Región Metropolitana. Sin embargo, aun cuando no se pueden generalizar sus resultados, la alarmante prevalencia de sintomatología en relación con el trabajo sugiere prestar atención a aspectos organizacionales tales como: las condiciones físico ambientales de trabajo, las relaciones sociales en el trabajo, la satisfacción percibida con la organización, las connotaciones cognitivas y emocionales de la experiencia de trabajo, el grado en que las políticas de la organización demandan acatamiento a los criterios de eficiencia y productividad propios de los estándares del mercado.

Así como estos datos nos hacen poner atención a elementos organizacionales de los lugares de trabajo de los profesionales estudiados, surge la pregunta por investigar en qué medida éstos son elementos estructurales del sistema de salud chileno. La sobrecarga de trabajo reportada y la sintomatología asociada, pudiesen comprenderse a la luz de un sistema que está sobrecargado, en tanto el sector público de salud chileno concentra la atención de los primeros cuatro quintiles de ingresos en quienes se constata un mayor riesgo y carga de enfermedad ${ }^{31}$ ".

\section{Referencias}

1. Almeida CM. Reforma do Estado e reforma de sistemas de saúde: experiências internacionais e tendências de mudança. Ciência \& Saúde Coletiva 1999; 4 (2): 263-86.

2. Homedes N, Ugalde A. Las reformas de salud neoliberales en América Latina: una visión crítica a través de dos estudios de caso. Rev Panam Salud Pública 2005; 17 (3): 210-20.

3. Blanch JM, Cantera L. El capitalismo sanitario como factor de riesgo psicosocial. En: Guillén C, Guil, R, coordinadores: Psicología Social: Un encuentro de Perspectivas. Cádiz: APPS 2007; p. 1075-80.

4. Jiménez JP. Bienestar y satisfacción de los médicos con el ejercicio de su profesión en un sistema de salud en proceso de reforma. Rev Med Chile 2004; 132: 635-36.

5. Jiménez J. Estrategias de los médicos para hacer frente a la crisis de la profesión. Rev Med Chile 2005; 133: 707-12.

6. Brito P. Desafíos y problemas de la gestión de recursos humanos de salud en las reformas sectoriales. Programa de Desarrollo de Recursos Humanos División de Desarrollo de Sistemas y Servicios de Salud. Observatorio de recursos humanos de salud. Washington: OPS/OMS; 2000.

7. Horwitz N. El Cambio de la práctica médica. Desafíos psicosociales para la profesión. Rev Med Chile 2004; 132 (6): 768-72.

8. Schonhaut L, Rojas P, Millán T. Calidad de vida de los médicos residentes del Programa de Formación de Especialistas en Pediatría. Rev Chil Pediatr 2009; 80 (1): 30-8.

9. Trucco M, Valenzuela P, Trucco D. Estrés ocupacional en personal de salud. Rev Med Chile 1999; 127 (12): 1453 61. 
10. Melita A, Cruz M, Merino J. Burnout en profesionales de enfermería que trabajan en centros asistenciales de la octava región, Chile. Cienc Enferm 2008; 14 (2): 75-85.

11. Astudillo P, Alarcón A, Lema M. Protectores de estrés laboral: percepción del personal de enfermería y médicos, Temuco, Chile. Cienc Enferm 2009; 15 (3): 111-22.

12. Avendaño C, Bustos P, Espinoza P, García F, Pierart T. Burnout y apoyo social en personal del servicio de psiquiatría de un hospital público. Cienc Enferm 2009; 15 (2): 55-68.

13. Piko B. Burnout, role conflict, job satisfaction and psychosocial health among Hungarian health care staff: A questionnaire survey. Int J Nurs Stud 2006; 43 (3): 31118.

14. Tyson P, Pongruengphant R. Five-year follow-up study of stress among nurses in public and private hospitals in Thailand. Int J Nurs Stud 2004; 41(3): 247-54.

15. Leguizamón L, Gómez V. Condiciones laborales y de salud en enfermeras de Santa Fe de Bogotá. IJCHP 2002; 2 (1): 173-82.

16. Thomas LS, Valli A. Levels of occupational stress in doctors working in a South African public-sector hospital. SAMJ 2006; 96 (11): 1162-68.

17. Blanch JM. El capitalismo organizacional como factor de riesgo psicosocial. Universidad Autónoma de Barcelona, Barcelona. Proyecto de investigación Referencia SEJ2007-63686/PSIC, Plan Nacional I+D+I (20082010), financiado por el Ministerio de Educación y Ciencia de España.

18. Blanch JM. Estudio de calidad de vida laboral en el subempleo. En: Romay J, Salgado J, Romaní M, Robla, D, compiladores: Psicología de las Organizaciones, del Trabajo, Recursos Humanos y de la Salud. Madrid: Biblioteca Nueva 2005; p 79-87.

19. Blanch JM (2003). La qualitat de vida laboral a l'Hospital. Bellaterra. UAB. (Informe de Evaluación. 82 p).

20. Blanch JM, Sahagún M, Cantera L, Cervantes G. Cuestionario de Bienestar Laboral General: Estructura y Propiedades Psicométricas. Questionnaire of General Labor Well-Being: Structure and Psychometric Properties. Revista de Psicología del Trabajo y las Organizaciones 2010; 26 (2): 157-170.

21. Blanch JM, Sahagún M, Cervantes G. Estructura factorial de la Escala de Condiciones de Trabajo. Revista de Psicología del Trabajo y las Organizaciones 2010; 26 (3): 175-89.
22. Grau A, Suñer R, García MM. Desgaste profesional en el personal sanitario y su relación con los factores personales y ambientales. Gaceta Sanitaria 2005; 19: 463-70.

23. Silveira-Roldán NN, Rodríguez-Carvajal R. El estrés laboral, la satisfacción laboral y el síndrome de Burnout en profesionales de la salud de Uruguay. En: Gil-Monte P, Moreno-Jiménez B, coordinadores: El síndrome de quemarse por el trabajo (Burnout). Grupos profesionales de riesgo. Madrid: Pirámide 2007; p. 171-86).

24. Muncer S, Taylor S, Green DW, McManus IC. Nurses' representation of the perceived causes ok work-related stress: a network drowing aproach. Work \& Stress 2001; 15 (1): 40-52.

25. Piko B. Psychosocial work environment and psychosomatic health of nurses in Hungary. Work and Stress 2003; 17 (1): 93-100.

26. Leda RM, Palomera A, Morante ME. El síndrome de Burnout en profesionales de enfermería mexicanos. En: Gil-Monte P, Moreno-Jiménez B, coordinadores: El síndrome de quemarse por el trabajo (Burnout). Grupos profesionales de riesgo. Madrid: Pirámide 2007; p. 163 70.

27. Rodríguez FJ, Blanco MA, Isaa S, Romero L, Gayoso P. Relación de la calidad de vida profesional y el Burnout en médicos de atención primaria. Atención Primaria 2005; 36: 442-47.

28. Benevides-Pereira AM. El síndrome de Burnout en Brasil y su expresión en el ámbito médico. En: Gil-Monte P, Moreno-Jiménez B, coordinadores: El síndrome de quemarse por el trabajo (Burnout). Grupos profesionales de riesgo. Madrid: Pirámide 2007; p. 187-201.

29. Bresó E, Salanova M, Schaufeli WB, Nogareda C. Síndrome de estar quemado por el trabajo "Burnout" (III): Instrumento de medición. Nota Técnica de Prevención,

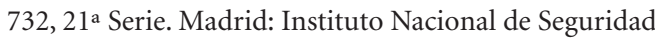
e Higiene en el Trabajo. 2007.

30. Gil-Monte P, Moreno-Jiménez B. Coordinadores. El sindrome de quemarse por el trabajo (Burnout). Grupos profesionales de riesgo. Madrid: Pirámide. 2007.

31. Cruces G. Protección social y sistemas de salud "Los sistemas de salud y de protección social frente a los nuevos escenarios epidemiológicos y demográficos”. Reunión de expertos sobre población y pobreza en América Latina y el Caribe. Organizado por la Comisión Económica para América Latina y el Caribe, CELADE-División de Población, con el auspicio del Fondo de Población de las Naciones Unidas (UNFPA). 2006. 